\title{
Considerações hidrodinâmicas sobre a derivação liquórica. Parte IV: Tecnologia de válvulas - Primeira geração
}

\author{
Angelo L. Maset ${ }^{1,2}$, José R. C. Pinto², José R. Andrade², Victor E. F. Xavier ${ }^{3}$
}

\section{RESUMO}

Sistemas valvulares para controle de hidrocefalia funcionam por meio de diferencial de pressão. As inovações tecnológicas surgidas após a primeira geração de válvulas tentam amenizar o desequilíbrio hidráulico causado pelo desvio artificial do liquor para fora da cavidade intracraniana causado pelo sistema valvular, e que agora sofrem com forças gravitacionais antes compensadas por mecanismos fisiológicos. Este trabalho esclarece ao neurocirurgião os parâmetros que qualificam o nível de desempenho das válvulas frequentemente utilizadas na prática neurocirúrgica, priorizando o entendimento do gráfico "pressão versus vazão". Para tal, os gráficos foram exemplificados por intermédio do teste de uma válvula de hidrocefalia de primeira geração disponibilizada recentemente, por meio dos testes da ISO 7197.

\section{PALAVRAS-CHAVE}

Hidrocefalia. Derivação ventriculoperitoneal.

\section{ABSTRACT}

Hydrodynamic considerations on CSF shunting. Part IV: technology of shunting valves Shunts for hydrocephalus work through a pressure differential. The technological innovations following $1^{\text {st }}$ generation shunts try to restore the hydrodynamic disequilibrium caused by the artificial CSF shift outwards the intracranial cavity caused by the shunt and that now suffer with gravitational forces formerly compensated by physiological mechanisms. This article describes to the neurosurgeon the parameters which qualify a shunt performance level of a device, giving priority to the pressure-flow relationship. To accomplish that, all ISO 7197 tests were exemplified using a $1^{\text {st }}$ generation shunt system recently introduced into the Brazilian market.

\section{KEY WORDS}

Hydrocephalus. Ventriculoperitoneal shunt.

\section{Introdução}

O conceito da diversão do liquor para outro compartimento corpóreo é antigo. Considera-se que a era moderna para os conjuntos para hidrocefalia tenha-se iniciado com Torkildsen, ${ }^{13}$ que utilizou novos materiais e descreveu a derivação dos ventrículos laterais para a cisterna magna em 1939. Matson ${ }^{11}$ introduziu a derivação lomboureteral em 1952 e Nulsen e Spitz ${ }^{12}$ introduziram o conceito da derivação ventrículo-jugular, bem como descreveram a primeira válvula com bola e mola, que foi popularizada mais tarde por Hakim. ${ }^{5}$ É creditado a Holter a introdução do silicone nos sistemas valvulares, o que trouxe uma melhoria significativa para todos os modelos de válvula pelo fato de o silicone ser muito bem tolerado pelo corpo humano. ${ }^{14}$
A derivação liquórica permanente é composta de três partes distintas, ou seja, um cateter proximal, que dá acesso ao sistema liquórico, a válvula, que impõe uma resistência adicional ao fluxo liquórico, e o cateter distal (Figura 1). Todos os sistemas de hidrocefalia funcionam por meio de um diferencial de pressão (DP) entre o cateter proximal (ventricular) e o cateter distal (mais comumente o peritoneal). Nosso artigo anterior ${ }^{10}$ abordou esse tema. Na verdade, todo sistema valvular funciona fisicamente como um conjunto de resistências em série, sendo que cada componente opõe alguma resistência ao fluxo liquórico (Figura 2), e a pressão da válvula, expressão conhecida pelo neurocirurgião, é a resultante (ou o somatório) das resistências de todos os componentes do sistema valvular.

1 Associação Portuguesa de Beneficência de São José do Rio Preto.

2 Laboratório de Pesquisa e Desenvolvimento de Ventura Biomédica Ltda.

3 Mestrando em Engenharia Mecânica (Ciências Térmicas) pela Universidade Estadual Paulista Júlio Mesquita Filho (Unesp). 


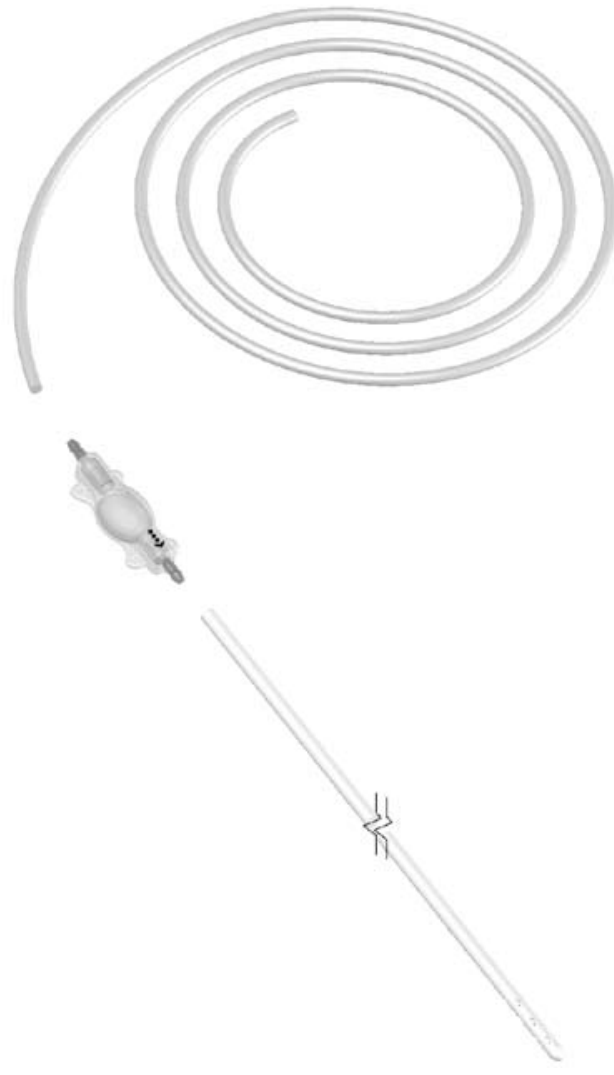

Figura 1 - Sistema valvular clássico.

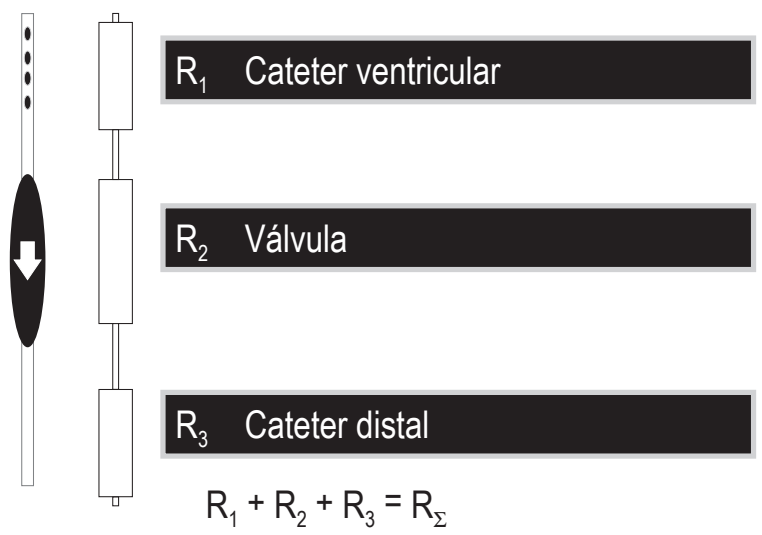

Figura 2 - Sistema valvular é visto fisicamente como resistências em série.

Na história do desenvolvimento das válvulas para hidrocefalia, a figura 2 representa o sistema para hidrocefalia mais básico. Todas as outras inovações, especificamente o mecanismo antissifão, o mecanismo com feedback negativo pressão-dependente (equivocadamente conhecidos como válvulas autorreguláveis ou fluxo dependente), o conceito de válvulas gravitacionais, as válvulas ajustáveis (ou programáveis, termo em desuso), com mecanismos magnéticos ou não, e finalmente a junção de válvulas ajustáveis com gravitacionais, fisicamente representam adições de resistências ao mecanismo básico concebido nos idos da década de 1960 e tentam amenizar o desequilíbrio hidrodinâmico causado pelo desvio artificial do liquor para fora da cavidade intracraniana e que agora sofrem com forças gravitacionais antes compensadas por mecanismos fisiológicos.

Existem critérios para a determinação da resistência a ser imposta pelo sistema valvular, e eles são determinados pela ISO 7197, edição revisada de 2006, denominada "Neurosurgical implants - Sterile, single-use hydrocephalus shunts and components". Esses critérios são seguidos pela maioria das indústrias. Todas elas devem obedecer aos mesmos critérios de fabricação mencionados anteriormente, mas o objeto de estudo deste trabalho serão as válvulas de diferencial de pressão básicas. O cateter distal influencia significativamente o desempenho de uma válvula, e será também objeto de estudo à parte. O objetivo deste estudo é apresentar os critérios da ISO 7197, os quais geram os gráficos de pressão versus vazão frequentemente apresentados aos neurocirurgiões, exemplificando-os por meio da apresentação dos resultados obtidos com uma nova válvula recentemente disponibilizada para utilização no mercado nacional.

\section{Materiais e métodos}

\section{Materiais}

A pressão gerada nos ventrículos cerebrais é transmitida por meio do sistema de condutos até ao elemento resistível da válvula (Figura 1). Existem vários tipos de elementos resistíveis. Os mais comuns são os modelos em fenda, modelo em membrana e modelo em bola e mola. A mola pode ser helicoidal ou reta. A figura 3 resume os elementos resistíveis mais comuns. $\mathrm{O}$ modelo em fenda (Figura 3a) utiliza as características do silicone, pois é um sulco realizado em uma estrutura cônica de silicone; dependendo do comprimento do sulco, esta estrutura opõe certa resistência à passagem do liquor.

O modelo em membrana (Figura 3b) é uma membrana de silicone com um eixo central; esse modelo atua por intermédio da mobilização das bordas da membrana, que cedem à pressão exercida pelos ventrículos e permite a drenagem.

O modelo em bola e mola (Figura 3c) é formado por um cilindro que aloja uma esfera de rubi e uma mola; a esfera e a mola estão apoiadas em lados opostos; 
EM REPOUSO

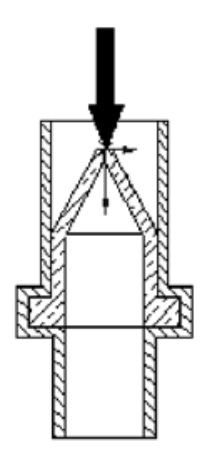

3a - Modelo em fenda

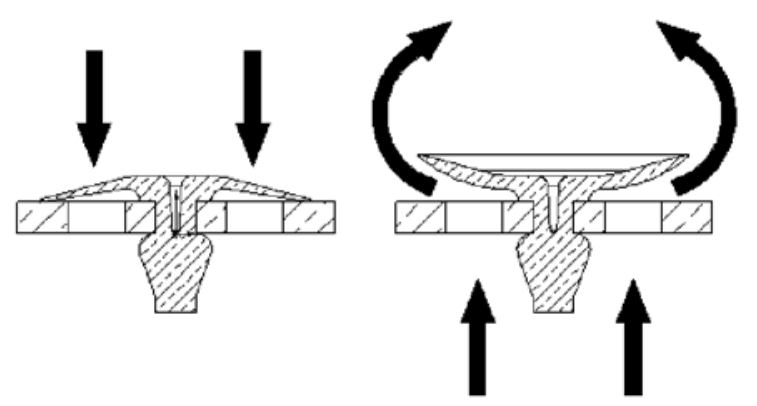

3b - Modelo em membrana
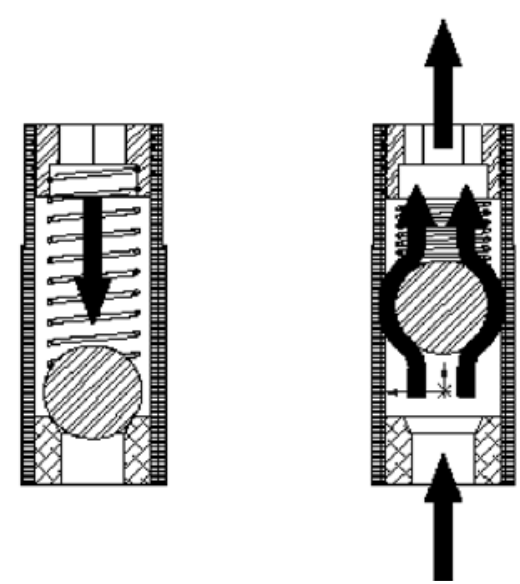

$3 c$ - Modelo em bola e mola

Figura 3 - Modelos de elementos resistivos.

conforme o liquor exerce pressão na bola, a mola cede e permite o fluxo liquórico no cilindro enclausurado no corpo de silicone pré-formado. Outro conceito de mola utiliza as molas lineares, que exercem pressão sobre a esfera que está alojada num berço. Conforme a pressão é exercida na esfera, no sentido de afastá-la do berço, ocorre a drenagem. As válvulas podem ser compostas por elementos resistivos únicos, duplos, ou mesmo híbridos, ou seja, podem ser compostas com diferentes mecanismos; não existe impedimento físico na utilização de diferentes elementos resistivos em uma única válvula. Qualquer que seja o mecanismo, eles atuarão como elementos resistivos em série.

A figura 4 é um desenho esquemático dos componentes essenciais de uma válvula em fenda, salientando a localização do elemento resistivo. Nessa figura, a válvula compõe-se de um corpo de silicone pré-formado (a), onde se inserem um conector de entrada (b) para conexão com o cateter proximal e um conector de saída (c) para conexão com o cateter distal. Os conectores de entrada e saída permitem a inserção de mecanismos resistivos que fazem a função valvular. Alternativamente, o mecanismo resistivo também pode ser incorporado ao corpo da válvula. $\mathrm{O}$ corpo da válvula também possui abaulamentos laterais e superiores que servem como câmeras ou reservatórios (d), que permitem testes de funcionalidade e do acesso ao liquor pelo neurocirurgião. Para a exemplificação dos testes da ISO 7197, foram selecionadas válvulas de primeira geração Synchrony - série Cello - com classificação "adulta" com as pressões de 20, 45, 70, 100 e $135 \mathrm{~mm}$ de $\mathrm{H}_{2} \mathrm{O}$ para análise de desempenho e segurança utilizando os testes normatizados. Utilizamos um cateter peritoneal de $120 \mathrm{~cm}$ de comprimento e $1,2 \mathrm{~mm}$ de diâmetro interno. Todos os testes foram realizados no Laboratório de Hidrodinâmica da empresa Ventura Biomédica e os resultados obtidos são apresentados a seguir.

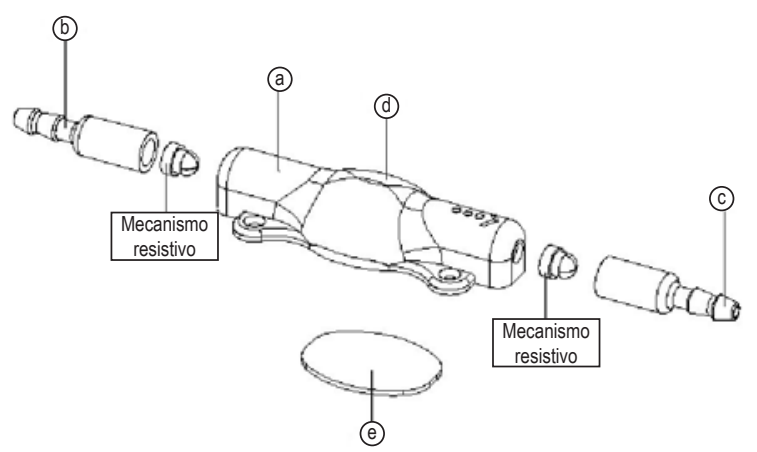

a) corpo de silicone; b) conector de entrada; c) conector de saída; d) câmara de compressão; e) base rígida

Figura 4 - Componentes essenciais de uma válvula em fenda.

\section{Métodos}

As válvulas para hidrocefalia devem ter o funcionamento e a confirmação da segurança do produto comprovados por meio de ensaios direcionados pela 
ISO 7197, edição revisada de 2006, denominada "Neurosurgical implants - Sterile, single-use hydrocephalus shunts and components", e se baseiam em seis tipos de ensaios, assim identificados:

1. Resistência a vazamentos

2. Resistência ao refluxo

3. Características de pressão versus vazão

4. Estabilidade a longo prazo

5. Resistência a pressões elevadas

6. Resistência à ruptura

Para a realização desses ensaios foram desenvolvidas e construídas bancadas de testes específicos. A seguir descrevem-se o conceito e a bancada para cada um desses testes:

1) Resistência a vazamentos: as válvulas devem suportar pressões muito superiores às pressões às quais trabalharão fisiologicamente; sendo assim, efetua-se o teste para verificação da ausência de vazamentos por intermédio da aplicação do ar nas válvulas até uma pressão equivalente a $1.000 \mathrm{~mm}$ de $\mathrm{H}_{2} \mathrm{O}$ durante cinco minutos. A não ocorrência de vazamentos determina a aprovação da válvula no citado teste, caso contrário, a válvula é reprovada e segregada dos demais testes para avaliação de desempenho. Para o ensaio é utilizada a bancada ilustrada na figura 5, que permite testar até cinco válvulas simultaneamente. A bancada é composta por uma seringa (a) responsável pela introdução do ar no sistema, acoplada em uma torneira de três vias (b) que permite a coleta de ar e o fechamento do sistema após a introdução da pressão desejada. $\mathrm{O}$ ar injetado é conduzido através de uma tubulação (c) até o reservatório hermeticamente fechado (d). A pressão imposta no sistema é verificada por meio do medidor de pressão (e) até que atinja o valor de $1.000 \mathrm{~mm}$ de $\mathrm{H}_{2} \mathrm{O}$ e pressurize os tubos (f) que conduzem $\mathrm{o}$ ar até as válvulas (g). As válvulas submersas em água bidestilada (h) presente no reservatório (i) são fixadas ao sistema de teste por meio dos conectores de entrada (j) e logo após as respectivas saídas possuem elementos para obstrução do ar denominados clamps (k). Ressalta-se que teste de válvulas em um número menor que cinco unidades é possível por intermédio do correto manuseio das torneiras de três vias (l) interpostas na entrada das tubulações que conduzem o ar até as válvulas.

2) Resistência ao refluxo: o shunt, além de permitir uma drenagem controlada do liquor, também deve impedir o retorno desse fluido para a cavidade ventricular durante a permanência

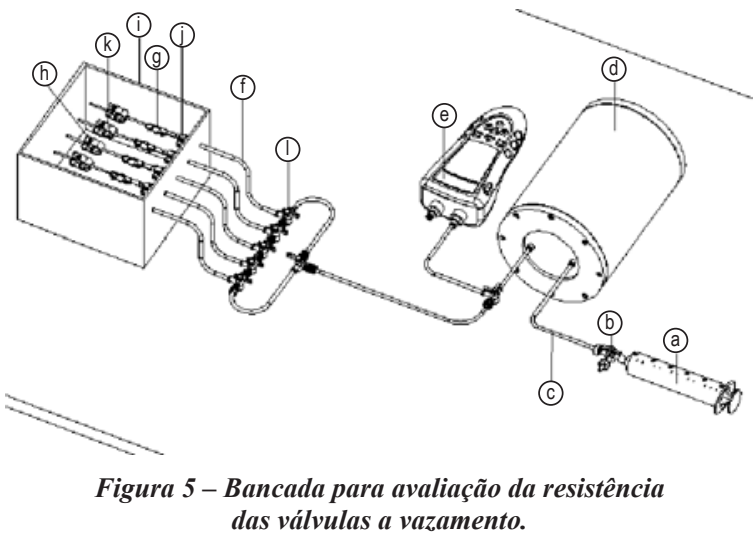

do indivíduo em posições corporais que possam facilitar essa ocorrência ou durante o teste clínico efetuado pelo neurocirurgião após o implante para avaliação do funcionamento do sistema. Para o atendimento dessa necessidade as válvulas adultas e pediátricas possuem instalada nos respectivos conectores de entrada uma válvula com fendas que permitem a passagem do escoamento do fluido conduzido para o cateter distal, mas impede o retorno desse fluido para o ventrículo por meio de seu fechamento quando o diferencial de pressão favorece seu retorno. Para a garantia da eficiência funcional da válvula quanto à resistência ao retorno do fluido ou refluxo, utiliza-se o aparato de teste ilustrado na figura 6. $\mathrm{O}$ teste consiste no preenchimento do sistema com água bidestilada utilizando uma seringa (a). O fluido passa através de uma torneira de três vias (b) e é conduzido pela tubagem (c) até a válvula (d) e logo após é direcionado à coluna graduada (e) sustentada pelo suporte (f). A válvula permanece submersa em água bidestilada (g) aquecida a $37 \pm 2{ }^{\circ} \mathrm{C}$ pela atuação de um aquecedor programável (h), ambos presentes no reservatório (i). A temperatura do fluido é verificada com o auxílio de um termômetro (j). As válvulas devem ser testadas quanto à resistência ao refluxo por intermédio da aplicação das pressões equivalentes a $100 \mathrm{~mm}$ de $\mathrm{H}_{2} \mathrm{O}$ e logo após de $500 \mathrm{~mm}$ de $\mathrm{H}_{2} \mathrm{O}$ e registradas na escala graduada. Para cada pressão aplicada avalia-se a resistência ao refluxo por meio do nível de água bidestilada presente na coluna graduada durante um intervalo de cinco minutos. A válvula é aprovada quando resiste ao refluxo quando o volume retornado for inferior a $0,2 \mathrm{~mL}$ durante os cinco minutos utilizados no teste em ambas as pressões aplicadas na saída da válvula. A não conformidade com os valores máximos de refluxo permitido, conforme norma ISO 7197, classifica a válvula como reprovada. 


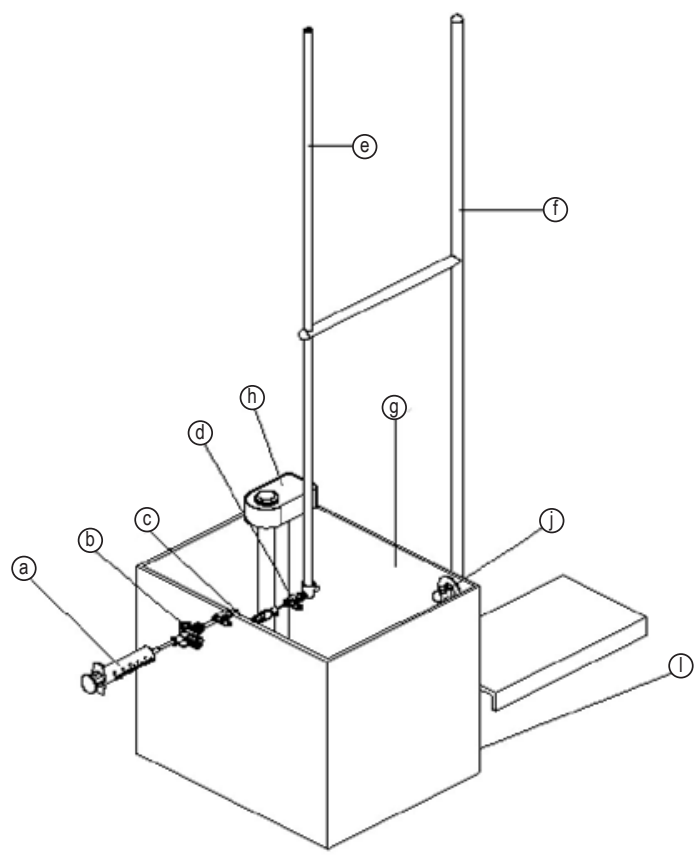

Figura 6-Bancada para avaliação da resistência das válvulas ao refluxo.

É importante salientar que a norma ISO 7197 não padroniza valores referenciais para válvulas ou seus limites superiores e inferiores. Cada fabricante determina o nível de desempenho de suas válvulas.

3) Características da pressão versus vazão: a pressão gerada nos ventrículos cerebrais é transmitida através do cateter ventricular para a válvula, que possui internamente elementos resistivos ao escoamento. É essencial a verificação in vitro do desempenho funcional de cada válvula utilizando-se a bancada de teste ilustrada na figura 7 .

O aparato para o teste das válvulas consiste de um reservatório de vidro (a) contendo água bidestilada (b) com temperatura de $37 \pm 2{ }^{\circ} \mathrm{C}$ pela atuação de um aquecedor programável (c) e verificada com o auxílio de um termômetro calibrado (d). O excesso de fluido presente no reservatório (a) é direcionado ao reservatório (e) e eliminado pelo dreno (f). Para a obtenção das variáveis (pressão e vazão) obtidas pelo teste é utilizada uma bomba de infusão programável (g) que succiona a água bidestilada presente em um reservatório denominado "Frasco de Mariotte" (h) que permite a manutenção da pressão interna constante mesmo com a saída do fluido presente em seu interior. A manutenção da pressão constante interna ao reservatório elimina a interferência causada no sistema

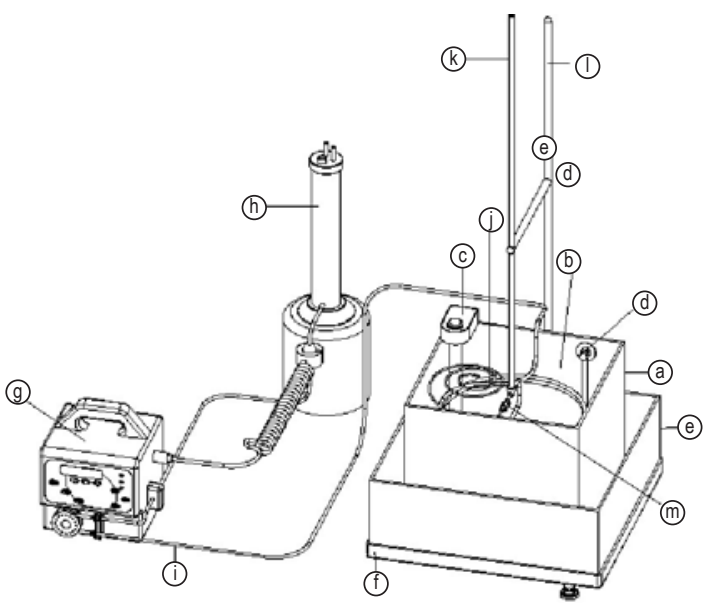

Figura 7-Bancada para determinação das características pressão $x$ vazão das válvulas.

caso a pressão do mencionado reservatório de alimentação (h) se altere conforme a redução do nível do fluido devido ao escoamento. O fluido é impulsionado pela bomba de infusão conforme cada vazão programada e é direcionado por intermédio de uma tubagem transparente (i) para facilitar a conferência da ausência de bolhas de ar no sistema. Nas proximidades do reservatório (a) a tubagem utilizada é de cobre (j) para a devida transferência de calor do fluido aquecido presente externo à tubagem e contido pelo reservatório (a) para o fluido escoado internamente ao tubo de cobre para garantir a temperatura do líquido equivalente a $37 \pm 2{ }^{\circ} \mathrm{C}$. O fluido aquecido conecta-se a um manómetro $(\mathrm{k})$ graduado com escala linear em mm, fixado por meio de um suporte (1) e é direcionado para a válvula testada $(\mathrm{m})$ e depois lançado no reservatório (a). A válvula deve permanecer submersa no fluido aquecido com uma altura de $10 \mathrm{~mm}$ entre o nível do fluido presente no reservátorio (a) e a base da válvula. Para o teste de cada válvula são programadas as vazões de 50, 40, 30, 20, 10 e $5 \mathrm{~mL} / \mathrm{h}$ no sistema por um período de cinco minutos para cada vazão, para a garantia da estabilidade do sistema. Coletam-se as respectivas pressões indicadas no manômetro $(\mathrm{k})$ por meio da coluna de fluido presente no interior desse tubo em cada vazão para cada válvula. Os valores obtidos são registrados e analisados por intermédio do gráfico de pressão versus vazão. Os valores obtidos devem encontrar-se dentro do limite superior de controle (LSC) e do limite inferior de controle (LIC) determinados para que a válvula seja aprovada, caso contrário, é reprovada. 
4) Estabilidade a longo prazo: para o teste do desempenho das válvulas a longo prazo foi desenvolvido o aparato de teste ilustrado na figura 8 , composto por um reservatório de vidro (a) contendo água bidestilada (b) e aquecida a uma temperatura de $37 \pm 2{ }^{\circ} \mathrm{C}$ pela atuação de um aquecedor (c) e constantemente em circulação dentro do reservatório pelo trabalho de uma bomba de circulação (d) responsável pela captação do fluido na parte do fundo do reservatório (e) e bombeamento através de um duto (f) para a região frontal do reservatório (g). As válvulas (h) são instaladas submersas ao fluido e com $10 \mathrm{~mm}$ de distância entre as respectivas bases e a superfície da água bidestilada. Uma bomba de membrana (i) é programada para fornecer vazões de $20 \mathrm{~mL} / \mathrm{h}$ para cada válvula testada com frequência de $60 \mathrm{~Hz}$, coletando a água bidestilada do reservatório interno (j) e conduzindo-a através da tubulação (k) até o tubo trocador de calor (1) que possibilita que a água bidestilada ingresse nas válvulas testadas com a mesma temperatura do banho. Cada válvula possui na entrada uma torneira de três vias $(\mathrm{m})$ que direciona o fluido para um manômetro graduado (n) fixado por um suporte (o).

As válvulas foram instaladas no aparato de teste para a determinação da estabilidade funcional. Após 15 dias de trabalho foram retiradas e testadas para a determinação das características de pressão versus vazão; logo após, foram instaladas no aparato para a verificação da instabilidade funcional a longo prazo até totalizar 28 dias de ensaios, e a seguir foram retiradas e testadas novamente para a determinação das características pressão versus vazão.

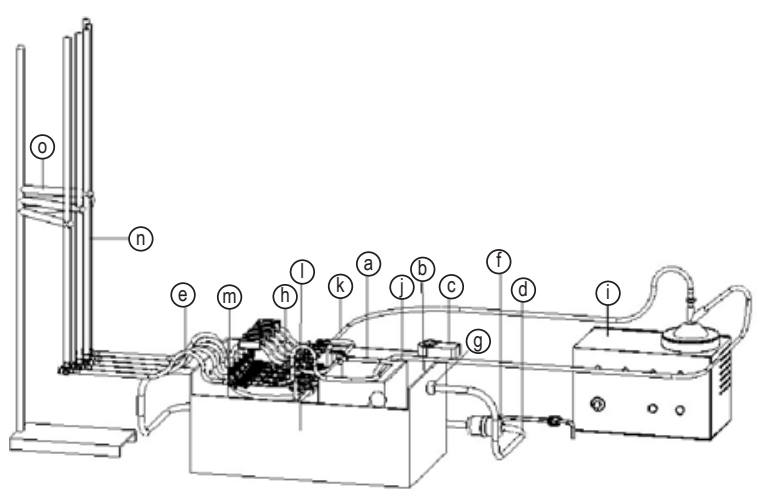

Figura 8 - Aparato para avaliação do desempenho das válvulas a longo prazo.
5) Resistência a pressão de $2.000 \mathrm{~mm}$ de $\mathrm{H}_{2} \mathrm{O}$ : cada componente da válvula deve suportar uma pressão equivalente a $2.000 \mathrm{~mm}$ de $\mathrm{H}_{2} \mathrm{O}$ durante um período de duas horas e apresentar variações dimensionais de no máximo $\pm 10 \%$ dos valores de referência. Para o ensaio utiliza-se o aparato indicado na figura 5 e o fluido de trabalho é a água bidestilada.

6) Resistência à ruptura: este teste avalia a resistência das válvulas diante de ruptura devida a esforços cíclicos. Para tal, foi construído o aparato de teste indicado na figura 9. Este teste determina a aplicação de esforços de tração nas válvulas referentes ao valor que ocorrer primeiro entre o alongamento de $10 \%$ da válvula ou a aplicação de uma força máxima de $5 \mathrm{~N}$, durante 100.000 ciclos a uma frequência de $1 \pm 0,2 \mathrm{~Hz}$.

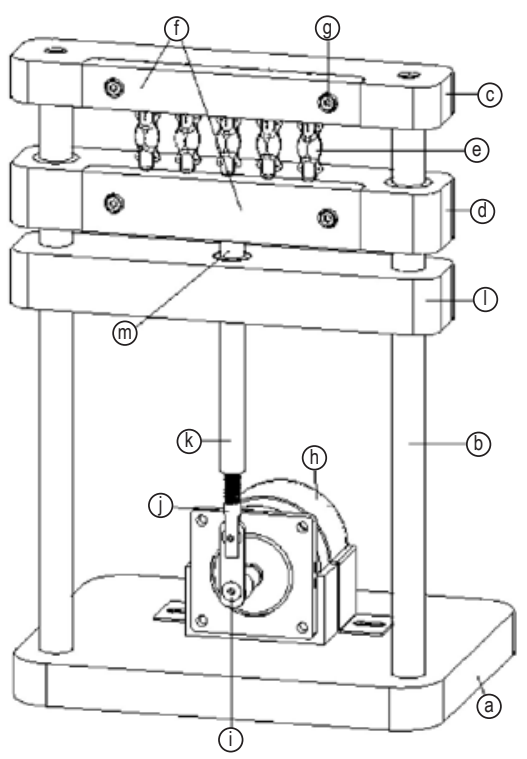

Figura 9 - Dispositivo para avaliação da resistência das válvulas á ruptura devido á esforços cíclicos.

$\mathrm{O}$ equipamento caracteriza-se por uma base rígida (a) que suporta duas colunas cilíndricas (b) que possuem fixadas na extremidade superior a base para fixação superior (c) e abaixo desta a base de fixação inferior (d). $\mathrm{O}$ aparato permite o teste de até cinco válvulas (e) fixadas nas bases superior e inferior por intermédio do suporte (f) que comprime a região externa dos conectores da válvula pelo ajuste dos parafusos $(\mathrm{g})$.

Acoplado à base encontra-se um motor elétrico (h) com um elemento excêntrico (i) disposto na extremidade do eixo, que movimenta a haste com rosca na extremidade (j) acoplada ao eixo guiado $(\mathrm{k})$ que passa através da base (1) e é suportado por um mancal de rolamento linear de esferas $(\mathrm{m})$ para a redução do 
atrito entre as partes. Ressalta-se que a base (1) pode ser ajustada conforme as dimensões lineares das válvulas testadas, devendo também ser ajustado o curso do elemento excêntrico e a haste roscada para as respectivas válvulas.

Para a determinação dos parâmetros ajustados no equipamento de teste, inicialmente foi avaliado o comportamento das válvulas para adultos testadas e constatou-se a ocorrência do alongamento de até 10\% antes da aplicação da força totalizando $5 \mathrm{~N}$. Esse procedimento foi efetuado com o auxílio de um dinamômetro digital marca Hiamada de precisão de 0,01 N e um paquímetro digital marca Mitutoyo com precisão de $0,01 \mathrm{~mm}$. Portanto, o equipamento de teste foi ajustado para o estiramento das válvulas ensaiadas equivalente ao alongamento de $10 \%$ de seu comprimento.

\section{Resultados}

\section{Resistência a vazamentos}

Após a aplicação do ar no interior das válvulas até a pressão de $1.000 \mathrm{~mm}$ de $\mathrm{H}_{2} \mathrm{O}$ constatou-se a não ocorrência de vazamentos e consequentemente a aprovação das válvulas ensaiadas.

\section{Resistência ao refluxo}

Os valores registrados indicam que todas as válvulas testadas apresentaram valores inferiores ao limite de controle de $0,4 \mathrm{~mL}$ durante o intervalo de cinco minutos de teste indicado pela norma ISO 7197, e, portanto, aprovadas no teste de resistência ao retorno do fluido. A maior parte das válvulas apresentou ausência de refluxo ou valores muito reduzidos. Os valores obtidos são apresentados na figura 10 .

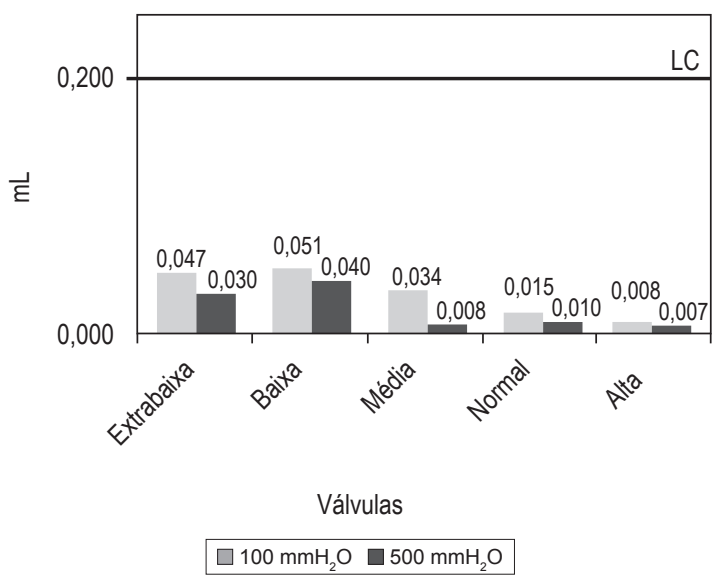

Figura 10 - Resultados dos testes para a avaliação de refluxo nas válvulas.

\section{Características da pressão versus vazão}

Os valores da média estão ilustrados na figura 11 . Esses mesmos valores e respectivos desvios-padrões estão registrados na tabela 1 . Como pode se observar por esta, as válvulas testadas apresentaram reduzidos desvios-padrões, indicando adequada reprodutibilidade dos valores.

\section{Estabilidade a longo prazo}

Por intermédio da análise dos resultados obtidos verifica-se que mesmo após 28 dias de trabalho as válvulas apresentaram comportamento adequado e com variações dentro dos limites de tolerância definidos como aceitáveis, portanto, sendo aprovadas nesse ensaio específico. Ressalta-se que durante o referido intervalo de tempo sob a influência da vazão de $20 \mathrm{~mL} / \mathrm{h}$ as pressões apresentadas nos manômetros não indicaram variações consideráveis e demonstraram boa estabilidade no funcionamento das válvulas. Os resultados obtidos são apresentados na figura 12.

\section{Resistência à pressão de $2.000 \mathrm{~mm}$ de $\mathrm{H}_{2} \mathrm{O}$}

Utilizando o aparato de teste indicado na figura 5, as válvulas testadas quanto à resistência à pressão de $2.000 \mathrm{~mm} \mathrm{H}_{2} \mathrm{O}$ foram aprovadas no referido teste.

\section{Resistência à ruptura}

Após a aplicação de 100.000 ciclos, todas as válvulas testadas no dispositivo indicado na figura 9 não apresentaram a ocorrência de rupturas e, portanto, foram aprovadas no teste de resistência à ruptura.

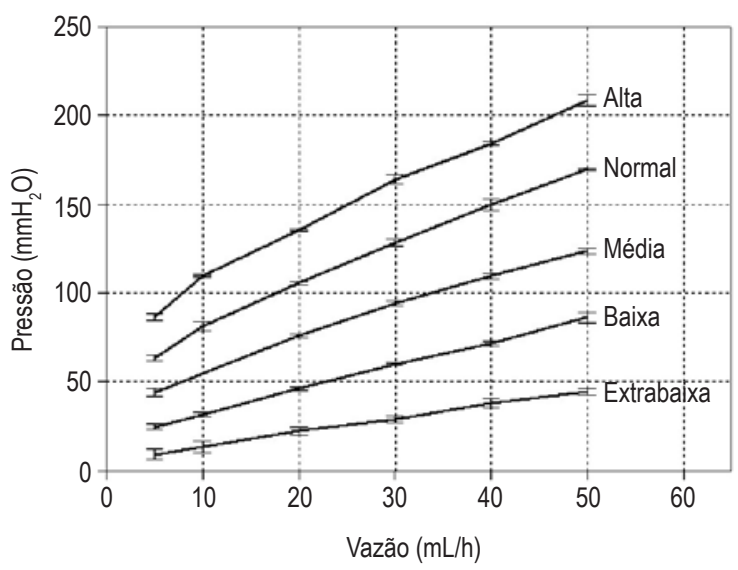

Figura 11 - Resultados das médias e dos desvios-padrões de cada classificação das válvulas. 
Tabela 1

Valores das pressões obtidas em função das vazões impostas juntamente às respectivas médias e aos desvios-padrões

\begin{tabular}{|c|c|c|c|c|c|c|c|}
\hline \multicolumn{8}{|c|}{ Perfil hidrodinâmico obtido com elementos resistivos ventura } \\
\hline \multicolumn{8}{|c|}{ Resistência a $20 \mathrm{mmH}_{2} \mathrm{O}$} \\
\hline Vazão (mL/h) & $\begin{array}{c}\text { Pressão } \\
\left(\mathrm{mmH}_{2} \mathrm{O}\right)\end{array}$ & $\begin{array}{c}\text { Pressão } \\
\left(\mathrm{mmH}_{2} \mathrm{O}\right)\end{array}$ & $\begin{array}{c}\text { Pressão } \\
\left(\mathrm{mmH}_{2} \mathrm{O}\right)\end{array}$ & $\begin{array}{c}\text { Pressão } \\
\left(\mathrm{mmH}_{2} \mathrm{O}\right)\end{array}$ & $\begin{array}{c}\text { Pressão } \\
\left(\mathrm{mmH}_{2} \mathrm{O}\right)\end{array}$ & $\begin{array}{c}\text { Média de pressão } \\
\left(\mathrm{mmH}_{2} \mathrm{O}\right)\end{array}$ & $\begin{array}{c}\text { Desvio-padrão } \\
\text { Pressão }\left(\mathrm{mmH}_{2} \mathrm{O}\right)\end{array}$ \\
\hline 50 & 45,00 & 42,00 & 42,00 & 46,00 & 46,00 & 44,20 & 2,05 \\
\hline 40 & 40,00 & 35,00 & 35,00 & 38,00 & 40,00 & 37,60 & 2,51 \\
\hline 30 & 30,00 & 27,00 & 27,00 & 31,00 & 30,00 & 29,00 & 1,87 \\
\hline 20 & 23,00 & 20,00 & 20,00 & 25,00 & 23,00 & 22,20 & 2,17 \\
\hline 10 & 15,00 & 10,00 & 10,00 & 17,00 & 15,00 & 13,40 & 3,21 \\
\hline 5 & 6,00 & 8,00 & 8,00 & 13,00 & 10,00 & 9,00 & 2,65 \\
\hline \multicolumn{8}{|c|}{ Resistência a $40 \mathrm{mmH}_{2} \mathrm{O}$} \\
\hline Vazão (mL/h) & $\begin{array}{c}\text { Pressão } \\
\left(\mathrm{mmH}_{2} \mathrm{O}\right)\end{array}$ & $\begin{array}{c}\text { Pressão } \\
\left(\mathrm{mmH}_{2} \mathrm{O}\right)\end{array}$ & $\begin{array}{c}\text { Pressão } \\
\left(\mathrm{mmH}_{2} \mathrm{O}\right)\end{array}$ & $\begin{array}{c}\text { Pressão } \\
\left(\mathrm{mmH}_{2} \mathrm{O}\right)\end{array}$ & $\begin{array}{c}\text { Pressão } \\
\left(\mathrm{mmH}_{2} \mathrm{O}\right)\end{array}$ & $\begin{array}{c}\text { Média de pressão } \\
\left(\mathrm{mmH}_{2} \mathrm{O}\right)\end{array}$ & $\begin{array}{c}\text { Desvio-padrão } \\
\text { Pressão }\left(\mathrm{mmH}_{2} \mathrm{O}\right)\end{array}$ \\
\hline 50 & 91,00 & 84,00 & 85,00 & 85,00 & 86,00 & 86,20 & 2,77 \\
\hline 40 & 70,00 & 73,00 & 71,00 & 71,00 & 72,00 & 71,40 & 1,14 \\
\hline 30 & 59,00 & 60,00 & 61,00 & 60,00 & 60,00 & 60,00 & 0,71 \\
\hline 20 & 45,00 & 46,00 & 47,00 & 45,00 & 47,00 & 46,00 & 1,00 \\
\hline 10 & 30,00 & 30,00 & 32,00 & 31,00 & 33,00 & 31,20 & 1,30 \\
\hline 5 & 24,00 & 22,00 & 26,00 & 24,00 & 26,00 & 24,40 & 1,67 \\
\hline \multicolumn{8}{|c|}{ Resistência a $70 \mathrm{mmH}_{2} \mathrm{O}$} \\
\hline Vazão (mL/h) & $\begin{array}{c}\text { Pressão } \\
\left(\mathrm{mmH}_{2} \mathrm{O}\right)\end{array}$ & $\begin{array}{c}\text { Pressão } \\
\left(\mathrm{mmH}_{2} \mathrm{O}\right)\end{array}$ & $\begin{array}{c}\text { Pressão } \\
\left(\mathrm{mmH}_{2} \mathrm{O}\right)\end{array}$ & $\begin{array}{c}\text { Pressão } \\
\left(\mathrm{mmH}_{2} \mathrm{O}\right)\end{array}$ & $\begin{array}{c}\text { Pressão } \\
\left(\mathrm{mmH}_{2} \mathrm{O}\right)\end{array}$ & $\begin{array}{c}\text { Média de pressão } \\
\left(\mathrm{mmH}_{2} \mathrm{O}\right)\end{array}$ & $\begin{array}{c}\text { Desvio-padrão } \\
\text { Pressão }\left(\mathrm{mmH}_{2} \mathrm{O}\right)\end{array}$ \\
\hline 50 & 122,00 & 123,00 & 125,00 & 125,00 & 123,00 & 123,60 & 1,34 \\
\hline 40 & 107,00 & 110,00 & 110,00 & 110,00 & 110,00 & 109,40 & 1,34 \\
\hline 30 & 92,00 & 95,00 & 95,00 & 95,00 & 95,00 & 94,40 & 1,34 \\
\hline 20 & 75,00 & 75,00 & 76,00 & 77,00 & 77,00 & 76,00 & 1,00 \\
\hline 10 & 55,00 & 55,00 & 55,00 & 55,00 & 55,00 & 55,00 & 0,00 \\
\hline 5 & 44,00 & 45,00 & 45,00 & 40,00 & 45,00 & 43,80 & 2,17 \\
\hline \multicolumn{8}{|c|}{ Resistência a $100 \mathrm{mmH}_{2} \mathrm{O}$} \\
\hline Vazão (mL/h) & $\begin{array}{c}\text { Pressão } \\
\left(\mathrm{mmH}_{2} \mathrm{O}\right)\end{array}$ & $\begin{array}{c}\text { Pressão } \\
\left(\mathrm{mmH}_{2} \mathrm{O}\right)\end{array}$ & $\begin{array}{c}\text { Pressão } \\
\left(\mathrm{mmH}_{2} \mathrm{O}\right)\end{array}$ & $\begin{array}{c}\text { Pressão } \\
\left(\mathrm{mmH}_{2} \mathrm{O}\right)\end{array}$ & $\begin{array}{c}\text { Pressão } \\
\left(\mathrm{mmH}_{2} \mathrm{O}\right)\end{array}$ & $\begin{array}{l}\text { Média de pressão } \\
\left(\mathrm{mmH}_{2} \mathrm{O}\right)\end{array}$ & $\begin{array}{c}\text { Desvio-padrão } \\
\text { Pressão }\left(\mathrm{mmH}_{2} \mathrm{O}\right)\end{array}$ \\
\hline 50 & 169,00 & 170,00 & 170,00 & 170,00 & 170,00 & 169,80 & 0,45 \\
\hline 40 & 149,00 & 152,00 & 150,00 & 145,00 & 153,00 & 149,80 & 3,11 \\
\hline 30 & 129,00 & 130,00 & 129,00 & 125,00 & 130,00 & 128,60 & 2,07 \\
\hline 20 & 105,00 & 107,00 & 105,00 & 105,00 & 106,00 & 105,60 & 0,89 \\
\hline 10 & 79,00 & 81,00 & 84,00 & 79,00 & 84,00 & 81,40 & 2,51 \\
\hline 5 & 64,00 & 64,00 & 64,00 & 64,00 & 61,00 & 63,40 & 1,34 \\
\hline \multicolumn{8}{|c|}{ Resistência a $135 \mathrm{mmH}_{2} \mathrm{O}$} \\
\hline Vazão (mL/h) & $\begin{array}{c}\text { Pressão } \\
\left(\mathrm{mmH}_{2} \mathrm{O}\right)\end{array}$ & $\begin{array}{c}\text { Pressão } \\
\left(\mathrm{mmH}_{2} \mathrm{O}\right)\end{array}$ & $\begin{array}{c}\text { Pressão } \\
\left(\mathrm{mmH}_{2} \mathrm{O}\right)\end{array}$ & $\begin{array}{c}\text { Pressão } \\
\left(\mathrm{mmH}_{2} \mathrm{O}\right)\end{array}$ & $\begin{array}{c}\text { Pressão } \\
\left(\mathrm{mmH}_{2} \mathrm{O}\right)\end{array}$ & $\begin{array}{l}\text { Média de pressão } \\
\left(\mathrm{mmH}_{2} \mathrm{O}\right)\end{array}$ & $\begin{array}{c}\text { Desvio-padrão } \\
\text { Pressão }\left(\mathrm{mmH}_{2} \mathrm{O}\right)\end{array}$ \\
\hline 50 & 210,00 & 210,00 & 212,00 & 205,00 & 205,00 & 208,40 & 3,21 \\
\hline 40 & 185,00 & 183,00 & 183,00 & 185,00 & 185,00 & 184,20 & 1,10 \\
\hline 30 & 163,00 & 160,00 & 167,00 & 164,00 & 165,00 & 163,80 & 2,59 \\
\hline 20 & 135,00 & 135,00 & 135,00 & 136,00 & 136,00 & 135,40 & 0,55 \\
\hline 10 & 110,00 & 110,00 & 109,00 & 110,00 & 109,00 & 109,60 & 0,55 \\
\hline 5 & 86,00 & 84,00 & 88,00 & 88,00 & 85,00 & 86,20 & 1,79 \\
\hline
\end{tabular}




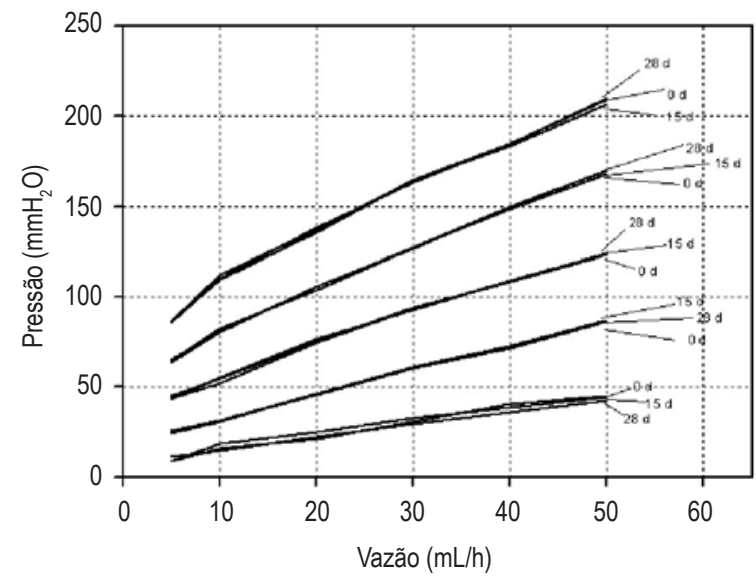

Figura 12 - Resultados dos ensaios de estabilidade a longo prazo.

\section{Discussão}

Existem diversos fatores físicos envolvidos na drenagem liquórica, como o diferencial de pressão entre as extremidades do cateter ventricular e peritoneal, a posição do paciente, o diâmetro e o comprimento dos tubos, a viscosidade. Esses conceitos podem ser revisados em nosso trabalho de $2005 .^{10}$

A apresentação do desempenho de cada válvula produzida é essencial, pois retrata o funcionamento relacionado com os parâmetros da pressão intraventricular e a vazão do liquor. Fabricantes de válvulas neurocirúrgicas apresentam o desempenho de seus produtos de diversas formas, desde a publicação do intervalo da possível variação da pressão sob a influência de uma vazão até intervalos de variação da pressão para cada vazão no intervalo de 5 a $50 \mathrm{~mL} / \mathrm{h}$. Ressalta-se também que os fabricantes dispõem diferentes classificações de válvulas e número variáveis de dispositivos que integram cada família de produto.

O desempenho das válvulas produzidas pela Ventura Biomédica, apresentado na figura 12, está disposto para as opções de produtos para pressões extrabaixa, baixa, média, normal e alta. Esse gráfico de pressão versus vazão (fluxo), tão familiar ao neurocirurgião, é a síntese do comportamento hidrodinâmico do sistema de derivação que o neurocirurgião pretende utilizar. Ele deveria ser interpretado da seguinte maneira: no eixo das abcissas existe a vazão imposta às válvulas, e no eixo das ordenadas existe a resistência imposta pela válvula, que gera uma pressão. Ao se impulsionar o fluido a uma uma vazão de $20 \mathrm{~mL} /$ hora pela bomba de infusão, por exemplo, para uma válvula de média pressão (resistência de 70 $\mathrm{mm}$ de $\mathrm{H}_{2} \mathrm{O}$ ), obtém-se uma média de 76,0 $\mathrm{mm}$ de $\mathrm{H}_{2} \mathrm{O}$ e desvio-padrão de $1,0 \mathrm{~mm}$ de $\mathrm{H}_{2} \mathrm{O}$ (Tabela 1 ).
$\mathrm{O}$ desvio máximo foi de $3,21 \mathrm{~mm}$ de $\mathrm{H}_{2} \mathrm{O}$, ocorrido em regime de resistência de $20 \mathrm{~mm}$ de $\mathrm{H}_{2} \mathrm{O}$ e vazão de $10 \mathrm{~mL} / \mathrm{h}$ e resistência de $135 \mathrm{~mm}$ de $\mathrm{H}_{2} \mathrm{O}$ e vazão de $50 \mathrm{~mL} / \mathrm{h}$. Assim, com base nos resultados obtidos na bancada de testes, definiram-se as faixas operacionais aceitáveis de variação dos valores de pressões de $\pm 10 \mathrm{~mm}$ de $\mathrm{H}_{2} \mathrm{O}$ com referência à pressão média registrada sob a influência da vazão de $5 \mathrm{~mL} / \mathrm{h}$ e média das pressões obtidas nos testes sob a influência da vazão de $50 \mathrm{~mL} / \mathrm{h}$ para o controle de qualidade. A representação gráfica pode ser observada na figura 13. Por outro lado, outros fabricantes preferem demonstrar o perfil hidrodinâmico de cada modelo de válvula utilizando-se apenas da média dos valores. O gráfico da figura 13 seria então representado como a figura 12, ou figura 11, sem o desvio-padrão. A diferença é que o neurocirurgião perde a percepção dos limites operacionais inferiores e superiores quando o gráfico é apresentado como na figura 11.

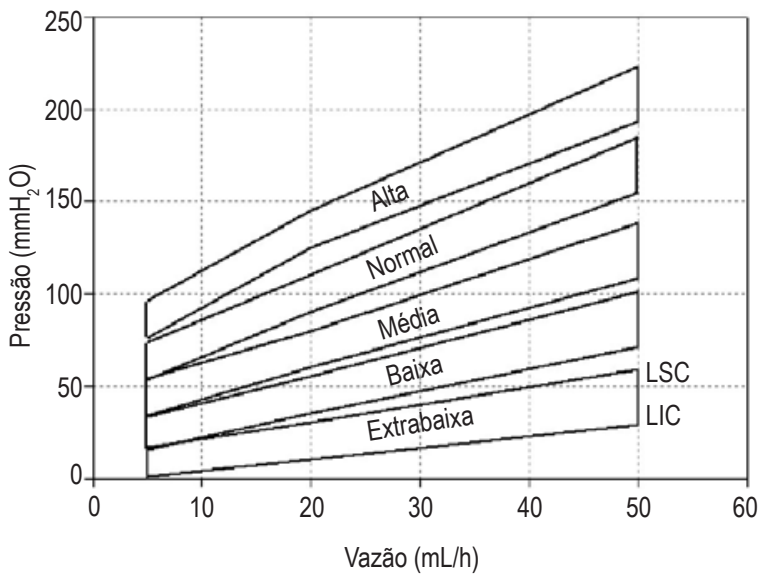

Figura 13 - Gráfico "pressão x vazão" determinado para as válvulas Ventura. Os limites inferiores e superiores de desempenho são estabelecidos pelo fabricante com base nos resultados de bancada. Quanto mais rigoroso o controle e a qualidade aplicados pelo fabricante menor é a faixa de variação aceitável - LIC (limite inferior de controle) $e$ LSC (limite superior de controle).

Como já se mencionou neste artigo, a mesma ISO 7197 não determina como uma válvula de determinada pressão deve desempenhar-se; ela apenas estabelece como os testes devem ser realizados. A resultante disso é que frequentemente existe uma interposição de desempenho de válvulas entre os vários fabricantes. Uma válvula de baixa pressão para um determinado fabricante pode eventualmente trabalhar como uma válvula de média pressão dentro do diagrama de pressão de outro fabricante. 


\section{Conclusões}

O gráfico pressão versus vazão é frequentemente apresentado ao neurocirurgião como um indicador de desempenho da válvula, mas também é fato que ele é frequentemente incompreendido.

Este trabalho procurou esclarecer ao neurocirurgião os parâmetros que qualificam o nível de desempenho das válvulas frequentemente utilizadas na prática neurocirúrgica, priorizando o entendimento ao gráfico pressão versus vazão.

Esses dados foram exemplificados com os dados de bancada de uma nova válvula nacional de primeira geração, Synchrony - série Cello - com classificação "adulta", submetida para registro junto à Agência Nacional de Vigilância Sanitária (Anvisa). Conforme pode ser depreendido, respeitando-se as condições de fabricação propostas naquele relatório técnico, e com nossa experiência acumulada em hidrodinâmica liquórica, ${ }^{1-4,6-10}$ as válvulas testadas estiveram no mesmo nível das melhores válvulas importadas.

Além disso, detalhamos os equipamentos de bancada utilizados, todos dentro da regulamentação ISO 7197, para que essa informação possa eventualmente ser útil para algum serviço neurocirúrgico que queira produzir trabalhos científicos em hidrodinâmica.

\section{Referências}

1. Camilo Pinto JR. Simulação hidrodinâmica e caracterização experimental de mecanismos anti-sifão em sistemas de drenagem externa de líquido CEF alo. Tese de mestrado. Programa de Pós-Graduação em Engenharia Mecânica. Universidade Estadual Paulista. Campus Ilha Solteira, 2005.

2. Camilo Pinto JR, Maset AL, Duarte KP, Mansur SS, Vieira EDR. An apparatus for testing external shunts utilized in hydrocephalus surgery treatment. Arquivos do Congress of Thermal Sciences and Engineering 11. Curitiba, 2006.

3. Camilo Pinto JR, Maset AL, Duarte KP, Vieira DDR, Perini $E A$, Vieira EDR. Mechanical tests of tree-way valves of external neurosurgical shunts. Arquivos do Brazilian Conference on Dynamics, Control and their Applications - DINCON, 5. Guaratinguetá, 2006.

4. Camilo Pinto JR, Maset AL, Mansur SS, Vieira EDR. Bancada para simulação hidrodinâmica de mecanismos anti-sifão em sistemas de drenagem externa do líquido cefalorraquidiano. IV Congresso Temático de Dinâmica, Controle e Aplicações. Bauru, 2005.

5. Hakim S, Venegas JG, Burton JD. The physics of cranial cavity, hydrocephalus and normal pressure hydrocephalus. Mechanical interpretation and mathematical model. Surg Neurol. 1976;5:187-210.

6. Maset AL, Camilo JR. O efeito sifão em pacientes com drenagem externa. Arquivos do VI Congresso da Sociedade Brasileira de Neurocirurgia Pediátrica. Belo Horizonte, 2005. p.48.

7. Maset AL, Camilo JR, Duarte KP, Vieira ER. Considerações hidrodinâmicas sobre a derivação liquórica. Parte III: Distúrbios hidrodinâmicos em sistemas de drenagem externa. Proposta de solução. Arq Bras Neurocir. 2006;25:100-11.

8. Maset AL, Camilo JR, Vieira DDR. Considerações hidrodinâmicas sobre a derivação liquórica. Parte II: $\mathrm{O}$ efeito sifão em sistemas de drenagem externa. Arq Bras Neurocir. 2005;24:45-51.

9. MasetAL, Camilo JR, Vieira EDR. "CSF dynamics in external drainage systems: the siphoning effect and possible role of anti-siphon devices". Hydrocephalus 2006, Proceedings. Sweden: Goteborg, 2006. p.74.

10. Maset AL, Castro SC, Camilo JR. Considerações hidrodinâmicas sobre a derivação liquórica. Parte I: Efeitos do cateter peritoneal. Arq Bras Neurocir. 2005;24:9-16.

11. Matson DD. A new operation for the treatment of communicating hydrocephalus. J Neurosurg. 1949;6:238-47.

12. Nulsen FE, Spitz EB. Treatment of hydrocephalus by direct shunt from ventricle to jugular vein. Surg Forum. 1952;30:399-403.

13. Torkildsen A. A new palliative operation in cases of inoperable occlusion of the Sylvian aqueduct. Acta Chir Scand. 1939;82:117-24.

14. Wallman LJ. Shunting for hydrocephalus. An oral history. Neurosurgery. 1982;11:308-13.

Original recebido em março de 2009

Aceito para publicação em junho de 2009

Endereço para correspondência

Angelo Luiz Maset

Av. Anísio Haddad, 7.700

15090-190 - São José do Rio Preto, SP

E-mail:maset@terra.com.br 